\title{
The role of bone morphogenetic proteins in liver fibrosis
}

\begin{abstract}
Liver fibrosis is featured by excessive accumulation of extracellular matrix (ECM) proteins in the injury liver of diseases, including alcoholic liver disease (ALD), non-alcoholic fatty liver disease (NAFLD) and steatohepatitis (NASH), cirrhosis, and hepatocellular cancer (HCC). However, there is no current therapy for preventing and reversing liver fibrosis. Transforming growth factor- $\beta 1$ (TGF- $\beta 1$ ) is a key profibrotic gene that drives activation of hepatic stellate cells (HSCs) and their transdifferentiation to myofibroblasts producing ECM proteins. Bone morphogenetic proteins (BMPs) belonging to TGF- $\beta$ big family play important roles in tissue morphogenesis and homeostasis in the bone, cartilage, muscle, kidney, and blood vessels. Accumulating evidence indicates that BMPs are involved in the development and progression of liver fibrosis and liver regeneration. In this review, we summarize the recent findings of the dual roles of BMPs in liver fibrosis. Strategies have been developed to target these molecules to treat liver fibrosis. However, the efficacy is under expectation. Therefore, better understanding of the underlying mechanisms of BMPs in the progression of liver disease is critically important for improving their therapeutic effect.
\end{abstract}

Keywords: liver fibrosis, bone morphogenetic proteins, transforming growth factor- $\beta$, hepatic stellate cells, therapy
Volume I2 Issue I - 202I

\author{
Ming Yang,' Chunye Zhang² \\ 'Department of Surgery, University of Missouri, Columbia, \\ Missouri, USA \\ ${ }^{2}$ Department of Veterinary Pathobiology, University of Missouri, \\ Columbia, Missouri, USA
}

\begin{abstract}
Correspondence: Ming Yang, Department of Surgery, University of Missouri, Columbia, Missouri, USA,
\end{abstract}

Email yangmi@health.missouri.edu

Received: November 30, 2020 | Published: February 12, 202
Abbreviations: ALD, alcoholic liver disease; ECM, extracellular matrix; NAFLD, non-alcoholic fatty liver disease; HCC, hepatocellular cancer; HSCs, hepatic stellate cells; EMT, epithelial-mesenchymal transition; CNS, central nervous system; BDL, bile duct ligation; MCD, methionine choline-deficient; HF, high-fat

\section{Introduction}

Liver fibrosis is featured by excessive accumulation of extracellular matrix proteins (ECMs) in the injury liver of many chronic liver diseases, including alcoholic liver disease (ALD), nonalcoholic fatty liver disease (NAFLD) and steatohepatitis (NASH), cirrhosis, and hepatocellular cancer (HCC).$^{1-3}$ The ECM producing cells are mainly from activated hepatic stellate cells (HSCs) or liver myofibroblast. ${ }^{4}$ In the liver, transforming growth factor- $\beta$ (TGF- $\beta$ ) signaling is implicated in liver disease progression, starting from liver inflammation and injury to liver fibrosis, cirrhosis, and final cancer. $^{2,5}$ TGF- $\beta$ is also the critical gene that drives the activation of HSCs and liver fibrosis. ${ }^{6}$ Bone morphogenetic proteins (BMPs) belonging to the TGF- $\beta$ big family play essential roles in tissue morphogenesis and homeostasis, including bone, cartilage, muscle, kidney, and blood vessels. ${ }^{7}$ Accumulating data indicate that BMPs are involved in the development and progression of liver fibrosis and liver regeneration. ${ }^{8}$ Vacca et al. ${ }^{9}$ reported that increased expression of BMP8B promoted the development of NASH both in animal models and human patients by activating HSCs via Smad-TGF- $\beta$ signaling. ${ }^{9}$ Furthermore, BMPs are also involved in the advanced stage of liver fibrosis, including cirrhosis and HCC. For instance, the expression of BMP9 was increased in tumor border samples of mouse HCC and human HCC tissue samples, which was associated with E-cadherin and Snail expression..$^{10}$ BMP9 also advanced the epithelial-mesenchymal transition (EMT) in HCC cell lines HLE and HepG2, as BMP9 induced cell migration. ${ }^{10}$ The effects of BMPs in tumor generation and progression have been well-reviewed in the previous publication. ${ }^{11}$ Herein, we summarize the recent findings of their roles in liver fibrosis.

\section{Bone morphogenetic proteins}

BMPs, a highly conserved family of regulator proteins, play important roles in homeostatic and pathological responses in all tissues and organs, such as the development of central nervous system $(\mathrm{CNS})^{), 12}$ cardiovascular disease, ${ }^{13}$ diabetes,${ }^{14}$ and autoimmune disease. ${ }^{15}$ BMPs transduce signals through type I and type II serine/threonine kinase receptors (a heterotetrameric complex) to downstream intracellular molecules, divided into canonical and non-canonical pathways. ${ }^{16}$ In the canonical signaling pathway, the effect of BMPs is mediated via the Smad-dependent pathway, while in the non-canonical pathway, the function of BMPs is mediated through non-Smad signaling. Targeting BMP provides a potential treatment. For example, obese leptin receptor-deficient $(\mathrm{db} / \mathrm{db})$ mice treated with BMP inhibitor LDN-193189, an inhibitor of BMP type I receptor signal transduction, decreased hepatic triglyceride levels. ${ }^{17}$ This function was associated with the reduced expression of diacylglycerol O-acyltransferase 2 (Dgat2), which is responsible for the conversion of diacylglycerol (DAG) to triglyceride. ${ }^{18}$

\section{BMPs with anti-fibrotic effects}

TGF- $\beta 1$ is an important pro-fibrotic gene contributing to the pathogenesis of liver fibrosis. ${ }^{6}$ As a TGF- $\beta$ family member, BMPs also play an essential role in liver fibrosis. Here, we discussed the function of each specific BMP in liver fibrosis.

Hepatic BMP2 is mainly expressed in the parenchymal hepatocytes and activated HSCs. BMP2 is reversely correlated with TGF- $\beta 1$ expression in human and mouse fibrotic livers. Adenovirusmediated BMP2 expression alleviated bile duct ligation (BDL) surgery or carbon tetrachloride $\left(\mathrm{CCl}_{4}\right)$ administration induced murine liver fibrosis, evidenced by the decrease of serum hepatic enzymes, cholangiocyte marker cytokeratin-19 (CK-19), and HSC activation markers. ${ }^{19}$ The mechanistic study showed that BMP2 inhibits TGF$\beta 1$-induced HSC activation, associated with attenuated expression of $\alpha$-smooth muscle actin ( $\alpha$-SMA) and fibronectin and reversed epithelial-to-mesenchymal transition markers, indicating that BMP2 
serves as a protective effect on liver fibrosis. ${ }^{19}$ Moreover, BMP2 as an angiocrine factor, was downregulated in Gata4-decifient liver sinusoidal endothelial cells (LSECs) that promoted liver fibrosis and HSC activation. ${ }^{20}$

BMP6 is a crucial regulator of iron homeostasis. In murine NAFLD, BMP6 expression was upregulated in hepatic steatosis, which was also shown in primary human hepatocytes with lipid accumulation. Methionine choline-deficient (MCD) and high-fat (HF) diets significantly increased the severity of liver inflammation and fibrosis in BMP6 $6^{-/-}$mice compared to wild-type mice. In vitro cell culture assay showed that treatment of recombinant BMP6 prevented the activation of HSCs and reduced the associated expression of proinflammatory and pro-fibrogenic genes, indicating a protective effect on liver fibrosis. ${ }^{21}$

In physiological conditions, BMP7 can improve insulin sensitivity in mouse muscle, adipose, and liver by potentiating PI3K/AKT pathway, resulting in decreased blood glucose levels. ${ }^{22}$ However, in HFD-induced insulin-resistant state, the expression BMP7 was decreased, and the above-mentioned positive effect was abrogated. Recombinant human bone morphogenic protein 7 (rhBMP7) has been shown to ameliorate renal fibrosis and to improve kidney function. In the porcine-serum peritoneal injection-induced rat liver fibrosis model, collagen production was reduced by rhBMP7 treatment via inhibiting the TGF- $\beta 1$ signaling pathway. ${ }^{23}$ BMP7 also downregulated Snailinduced mRNA and protein levels of $\alpha$-SMA and E-cadherin in rat liver epithelial cells compared to untreated cells. Furthermore, BMP7 induced mesenchymal-epithelial transition (MET) by Snail and Notch involved signaling pathway. ${ }^{24} \mathrm{In}$ the development of liver fibrosis both in the $\mathrm{CCl}_{4}$-induced murine model and human tissue, BMP7 protein expression first increased, and then decreased due to the inhibition by a sustainably increased TGF- $\beta 1$ protein expression. Besides, exogenous BMP7 inhibited the activation, migration, and proliferation of HSCs in vitro by selectively regulating the TGF- $\beta /$ Smad signaling pathway, and improved liver fibrosis in vivo, suggesting an anti-liver fibrosis function. ${ }^{25}$ Another in vivo study showed that intraperitoneal injection of human recombinant BMP7 decreased the expressions of TGF- $\beta 1$ and $\alpha$-SMA in ICR mouse liver and inhibited the expression of epidermal growth factor receptor (EGFR) and phosphorylatedepidermal growth factor receptor (pEGFR). ${ }^{26}$

\section{BMPs with pro-fibrotic effects}

Treatment with anti-BMP1-3, an isoform of BMP1, decreased $\mathrm{CCl}_{4}$-induced rat liver fibrosis, evidenced by downregulated ECMs (e.g., collagen type I) and decreased plasma levels of TGF- $\beta 1 .{ }^{27}$ In comparison to non-cancerous liver fibroblasts (NFs), BMP4 was found up-regulated in cancer-associated fibroblasts ( $\mathrm{CAFs}$ ), which expressed more ECM genes such as ACTA2 (smooth muscle actin alpha 2) and COL1A1 (alpha-1 type I collagen). In addition, overexpression of BMP4 in fibroblasts showed the phenotype and function of CAFs. The increased expression of BMP4 in resected tissues from human HCC patients was associated with reduced postoperative overall survival. ${ }^{28}$

BMP8B is a major contributor to NASH since it promotes the activation of HSCs via both $\operatorname{Smad} 2 / 3$ and Smad1/5/9 branches of the TGF $\beta$-BMP signaling pathway. BMP8B depletion prevented HSC activation and reduced inflammatory response, resulting in limitation of NASH progression. Evidence was also featured in the NASH model of primary human $3 \mathrm{D}$ microtissues when challenged with recombinant BMP8. ${ }^{9}$

In the liver, HSCs are the primary cells producing BMP9. Activating HSCs in vitro or in vivo increased BMP9 production, resulting in enhanced liver damage and inhibited proliferation in chronic or acute liver injury. ${ }^{29}$ Liver biopsy data also showed that increased expression of BMP9 was tested in patients with advanced liver fibrosis. Blocking BMP9 expression in mice either by adenovirus-mediated BMP9 knockdown or by monoclonal antibody successfully attenuated liver fibrosis. A molecular study showed that BMP9 overexpression activates HSCs through Smad-mediated or inhibiting DNA binding protein 1 (ID1) signaling pathway, resulting in the progression of liver fibrosis. These results suggest that BMP9 may serve as a diagnostic and therapeutic target for liver fibrosis. ${ }^{30}$

Furthermore, BMP9 plays an essential role in LSECs by binding its receptor activin receptor-like kinase 1 (ALK1). BMP9 deletion induces hepatic perisinusoidal fibrosis in 129/Ola mice with age more than 15 weeks. LSECs from BMP9 knockout mice reduced fenestration compared with those from wild-type mice, resulting from high expression of CD34 and low expression of several terminal differentiation markers, such as lymphatic vessel endothelial receptor 1 (Lyve1) and plasmalemma vesicle-associated protein (Plvap). Supplementation of BMP9 in cultured primary LSECs kept the expression levels of LSEC-specifying transcription factor GATAbinding protein 4 (Gata4) and Plvap, promoting the maintenance of LSEC fenestration. These results show that BMP9 controls LSEC fenestration and protects against perivascular hepatic fibrosis in 129/ Ola mice. ${ }^{31}$ However, in C57BL/6 mice, BMP9 depletion did not affect LSEC fenestration and differentiation markers' expression. The mRNA expression of LSEC differentiation markers, including Stabilin 2, Plvap, and CD209b, was significantly lower in wild-type C57BL/6 compared with wild-type 129/Ola mice, indicating a phenotype of capitalization of LSECs in C57BL/6 mice. In addition, the circulating concentration of BMP9 in C57BL/6 mice was lower compared with the 129 /Ola mice. ${ }^{32}$ These results suggest that the role of BMP9 in LSEC fenestration and hepatic fibrogenesis is dependent on genetic background. Overall, BMPs show different functions in liver fibrosis, which is summarized in Figure 1.

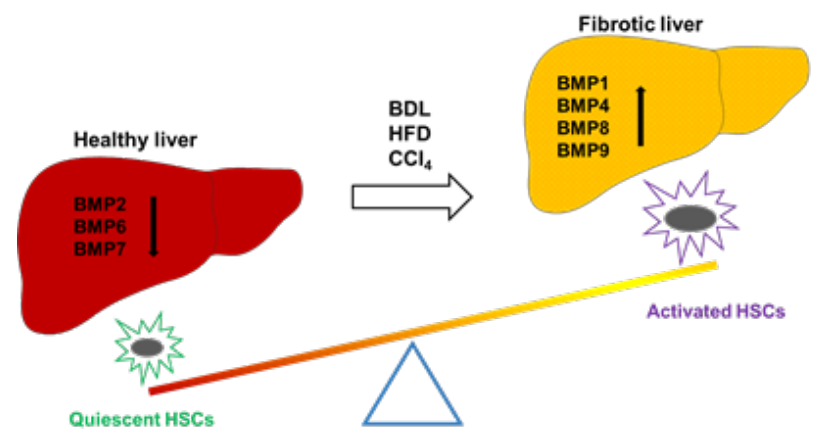

Figure I The role of BMPs in liver fibrosis.

Treatments, including bile duct ligation (BDL) surgery, carbon tetrachloride $\left(\mathrm{CCl}_{4}\right)$, and high-fat diets (HFD), promote the development of liver fibrosis. ${ }^{19,21}$ The expression of BMPs varies with liver fibrosis progression and activation of hepatic stellate cells (HSCs). BMP2, ${ }^{19}$ BMP6, ${ }^{21}$ and BMP7 ${ }^{25}$ have been shown to play antifibrotic effect, while BMP $1,{ }^{27} \mathrm{BMP} 4,{ }^{28} \mathrm{BMP} 8,{ }^{9}$ and $\mathrm{BMP} 9^{30}$ promote fibrogenesis. Therefore, downregulation of BMP2, 6 and 7 and overexpression BMP1, BMP4, BMP8, and BMP9 promote liver fibrosis.

\section{BMP-mediated treatment for liver fibrosis}

Advanced studies have been processed to investigate whether targeting these molecules could inhibit liver fibrosis (Table 1). 
For example, herbal compound 861 has been shown to attenuate liver fibrosis both in a BDL-induced liver fibrosis rat model and human patients, evidenced by histological and serum biochemical analysis. The molecular mechanism study showed that compound 861 signaling increased the expression of BMP7 and phospho (p)-Smad1/5/8 in hepatocytes of BDL-induced fibrotic liver. ${ }^{33}$ Another study showed that miR-542-3p can promote liver fibrosis by inhibiting BMP7 expression. Inhibition of miR-542-3p can prevent $\mathrm{HSC}$ activation and $\mathrm{CCl}_{4}$-induced liver fibrosis in mice via modulating $\alpha$-SMA, collagen, and TGF- $\beta$ expression. Further study showed that miR-542-3p promoted liver fibrosis by inhibiting BMP7 expression via directly binding to the 3'-untranslated region of BMP7 mRNA. ${ }^{34}$ Adenoviral-transduction with BMP7 treatment in hamsters significantly decreased $\alpha$-SMA-positive cells, the deposit of collagen type I, and tissue inhibitor of metalloproteinases (TIMP)-2 expression, and increased matrix metalloproteinase (MMP)-13 expression. ${ }^{35}$

Table I Targeting BMPs to treat liver fibrosis

\begin{tabular}{|c|c|c|c|}
\hline BMPs & Liver fibrosis model & Treatment & Reference \\
\hline BMPI-3 & $\begin{array}{l}\mathrm{CCl}_{4} \text {-induced rat liver } \\
\text { fibrosis }\end{array}$ & $\begin{array}{l}\text { Treatment with anti-BMPI-3 antibodies decreased the expression of collagen type I and } \\
\text { genes TgfbI, Itgb6, Colla I, and Acta2. }\end{array}$ & 27 \\
\hline BMP2 & $\begin{array}{l}\mathrm{BDL} \text { - and } \mathrm{CCl}_{4} \text {-induced } \\
\text { mouse liver fibrosis }\end{array}$ & $\begin{array}{l}\text { Adenovirus-mediated BMP2 gene delivery reduced } \alpha \text {-SMA expression in the BDL model, } \\
\text { suppressed fibronectin expression in the } \mathrm{CCl}_{4} \text { model, and alleviated serum alkaline } \\
\text { phosphatase (ALP) levels. }\end{array}$ & 36 \\
\hline BMP4 & $\begin{array}{l}\text { High-fat diet (HFD)- } \\
\text { induced mouse NAFLD }\end{array}$ & $\begin{array}{l}\text { Exogenous BMP4 inhibited hepatic steatosis, ameliorated serum triglyceride levels and } \\
\text { body weight, and alleviated NAFLD progression in vivo. }\end{array}$ & 37 \\
\hline BMP5 & $\begin{array}{l}\text { Western diet (WD)- } \\
\text { induced mouse NASH. }\end{array}$ & $\begin{array}{l}\text { Docosahexaenoic acid (DHA) supplementation attenuated the expression of BMP5, } \\
\text { which may inhibit NASH progression to HCC. }\end{array}$ & 38 \\
\hline BMP7 & $\begin{array}{l}\mathrm{CCl}_{4} \text {-induced mouse } \\
\text { liver cirrhosis }\end{array}$ & $\begin{array}{l}\text { The antisense of miR- } 22 \text { expression mediated by viruses significantly ameliorates liver } \\
\text { fibrosis and portal hypertension caused by } \mathrm{CCl}_{4} \text {, possibly through the upregulation of } \\
\text { BMP7. }\end{array}$ & 39 \\
\hline BMP8 & $\begin{array}{l}\text { Primary human 3D } \\
\text { microtissues modeling } \\
\text { NASH }\end{array}$ & $\begin{array}{l}\text { Recombinant BMP8 induced NASH model of primary human 3D culture. BMP8B } \\
\text { depletion in vivo prevented HSC activation and reduced inflammation, reducing NASH } \\
\text { progression. }\end{array}$ & 9 \\
\hline BMP9 & $\begin{array}{l}\mathrm{CCl}_{4} \text {-induced mouse } \\
\text { liver fibrosis }\end{array}$ & $\begin{array}{l}\text { Injection of an anti-Bmp9 inhibitory monoclonal antibody }(\mathrm{mAb}) \text { reduced collagen I and } \\
\alpha-S M A \text { expression. }\end{array}$ & 30 \\
\hline
\end{tabular}

\section{Summary}

Liver wound healing accompanies fibrosis, which is considered a protection mechanism in liver damage. However, uncontrolled liver fibrosis brings problems in response to persistently chronic liver inflammation and injury. BMPs, as a critical TGF- $\beta$ member, play critical roles in liver fibrosis and regeneration. In addition, BMPs regulate other signaling pathways to impact the development of diseases and treatment. For instance, BMP-activin receptor-like kinase 1 (ALK1)-transmembrane protein 100 (TMEM100) signaling plays a pivotal role in inflammation, cell apoptosis, and proliferation and metastasis of tumors. ${ }^{40}$ An increasing number of studies have demonstrated the functions of BMPs in liver fibrosis. However, BMPs play different roles in liver fibrosis, selectively targeting them poses a promising approach for liver fibrosis treatment.

\section{Acknowledgments}

M.Y. and C.Z. conceived and drafted the review, contributing equally.

\section{Funding}

This work was not supported by a grant.

\section{Conflicts of interest}

The authors declare no conflict of interest.

\section{References}

1. Tsuchida T, Friedman SL. Mechanisms of hepatic stellate cell activation. Nat Rev Gastroenterol Hepatol. 2017;14:397-411.
2. Zhang C, Yang M, Ericsson AC. Antimicrobial Peptides: Potential Application in Liver Cancer. Front Microbiol. 2019;10:1257.

3. Zhang C, Yang M, Ericsson AC. The Potential Gut Microbiota-Mediated Treatment Options for Liver Cancer. Front Oncol. 2020;10:524205.

4. Iwaisako K, Jiang C, Zhang M, et al. Origin of myofibroblasts in the fibrotic liver in mice. Proc Natl Acad Sci U S A. 2014;111(32):E3297E3305.

5. Fabregat I, Caballero-Díaz D. Transforming Growth Factor- $\beta$-Induced Cell Plasticity in Liver Fibrosis and Hepatocarcinogenesis. Front Oncol. 2018;8:357.

6. Dewidar B, Meyer C, Dooley S, et al. TGF- $\beta$ in Hepatic Stellate Cell Activation and Liver Fibrogenesis-Updated 2019. Cells. 2019;8(11):1419.

7. Katagiri T, Watabe T. Bone Morphogenetic Proteins. Cold Spring Harbor Perspectives in Biology. 2016;8:a021899.

8. Herrera B, Addante A, Sánchez A. BMP Signalling at the Crossroad of Liver Fibrosis and Regeneration. Int J Mol Sci. 2017;19.

9. Vacca M, Leslie J, Virtue S, et al. Bone morphogenetic protein 8B promotes the progression of non-alcoholic steatohepatitis. Nat Metab. 2020;2(6):514-531.

10. Li Q, Gu X, Weng H, et al. Bone morphogenetic protein-9 induces epithelial to mesenchymal transition in hepatocellular carcinoma cells. Cancer Sci. 2013;104:398-408.

11. Zhang L, Ye Y, Long X, et al. BMP signaling and its paradoxical effects in tumorigenesis and dissemination. Oncotarget. 2016;7(47):7820678218.

12. Hart CG, Karimi-Abdolrezaee S. Bone morphogenetic proteins: New insights into their roles and mechanisms in CNS development, pathology and repair. Exp Neurol. 2020:113455. 
13. Guihard P, Guo Y, Wu X, et al. Shaping Waves of Bone Morphogenetic Protein Inhibition During Vascular Growth. Circ Res. 2020;127:12881305 .

14. Herdenberg C, Mutie PM, Billing O, et al. LRIG proteins regulate lipid metabolism via BMP signaling and affect the risk of type 2 diabetes. Commun Biol. 2021;4(1):90.

15. Eixarch H, Calvo-Barreiro L, Costa C, et al. Inhibition of the BMP Signaling Pathway Ameliorated Established Clinical Symptoms of Experimental Autoimmune Encephalomyelitis. Neurotherapeutics. 2020;17(4):1988-2003.

16. Wang RN, Green J, Wang Z, et al. Bone Morphogenetic Protein (BMP) signaling in development and human diseases. Genes Dis. 2014;1:87105.

17. Thayer TE, Lino Cardenas CL, Martyn T, et al. The Role of Bone Morphogenetic Protein Signaling in Non-Alcoholic Fatty Liver Disease. Sci Rep. 2020;10(1):9831.

18. Yen CL, Stone SJ, Koliwad S, et al. Thematic review series: glycerolipids. DGAT enzymes and triacylglycerol biosynthesis. J Lipid Res. 2008;49(11):2283-2301.

19. Chung YH, Huang $\mathrm{YH}, \mathrm{Chu} \mathrm{TH}$, et al. BMP-2 restoration aids in recovery from liver fibrosis by attenuating TGF- $\beta 1$ signaling. Lab Invest. 2018;98(8):999-1013.

20. Winkler M, Staniczek T, Kürschner SW, et al. Endothelial GATA4 controls liver fibrosis and regeneration by preventing a pathogenic switch in angiocrine signaling. J Hepatol. 2021;74(2):380-393.

21. Arndt S, Wacker E, Dorn C, et al. Enhanced expression of BMP6 inhibits hepatic fibrosis in non-alcoholic fatty liver disease. Gut. 2015;64(6):973-981.

22. Chattopadhyay T, Singh RR, Gupta S, et al. Bone morphogenetic protein-7 (BMP-7) augments insulin sensitivity in mice with type II diabetes mellitus by potentiating PI3K/AKT pathway. Biofactors. 2017;43(2):195-209.

23. Zhong L, Wang $\mathrm{X}$, Wang $\mathrm{S}$, et al. The Anti-Fibrotic Effect of Bone Morphogenic Protein-7(BMP-7) on Liver Fibrosis. Int J Med Sci. 2013;10(4):441-450.

24. Bi WR, Jin CX, Xu GT, et al. Bone morphogenetic protein-7 regulates Snail signaling in carbon tetrachloride-induced fibrosis in the rat liver. Exp Ther Med. 2012;4(6):1022-1026.

25. Zou GL, Zuo S, Lu S, et al. Bone morphogenetic protein-7 represses hepatic stellate cell activation and liver fibrosis via regulation of TGF- $\beta$ / Smad signaling pathway. World J Gastroenterol. 2019;25(30):42224234 .

26. Wang LP, Dong JZ, Xiong LJ, et al. BMP-7 attenuates liver fibrosis via regulation of epidermal growth factor receptor. International Journal of Clinical and Experimental Pathology. 2014;7:3537-3547.
27. Grgurevic L, Erjavec I, Grgurevic I, et al. Systemic inhibition of BMP13 decreases progression of $\mathrm{CCl}(4)$-induced liver fibrosis in rats. Growth Factors. 2017;35:201-215.

28. Mano Y, Yoshio S, Shoji H, et al. Bone morphogenetic protein 4 provides cancer-supportive phenotypes to liver fibroblasts in patients with hepatocellular carcinoma. J Gastroenterol. 2019;54(11):1007-1018.

29. Breitkopf-Heinlein K, Meyer C, König C, et al. BMP-9 interferes with liver regeneration and promotes liver fibrosis. Gut. 2017;66(5):939-954.

30. Li P, Li Y, Zhu L, et al. Targeting secreted cytokine BMP9 gates the attenuation of hepatic fibrosis. Biochimica et Biophysica Acta (BBA) Molecular Basis of Disease. 2018;1864(3):709-720.

31. Desroches-Castan A, Tillet E, Ricard N, et al. Bone Morphogenetic Protein 9 Is a Paracrine Factor Controlling Liver Sinusoidal Endothelial Cell Fenestration and Protecting Against Hepatic Fibrosis. Hepatology. 2019;70(4):1392-1408.

32. Desroches-Castan A, Tillet E, Ricard N, et al. Differential Consequences of Bmp9 Deletion on Sinusoidal Endothelial Cell Differentiation and Liver Fibrosis in 129/Ola and C57BL/6 Mice. Cells. 2019;8.

33. Hou F, Liu R, Liu X, et al. Attenuation of liver fibrosis by herbal compound 861 via upregulation of BMP-7/Smad signaling in the bile duct ligation model rat. Mol Med Rep. 2016;13:4335-4342.

34. Ji F, Wang K, Zhang Y, et al. MiR-542-3p controls hepatic stellate cell activation and fibrosis via targeting BMP-7. J Cell Biochem. 2019;120(3):4573-4581.

35. Cervantes-Garcia D, Cuellar-Juarez AG, Borrego-Soto G, et al Adenoviral-bone morphogenetic protein-7 and/or doxazosin therapies promote the reversion of fibrosis/cirrhosis in a cirrhotic hamster model. Mol Med Rep. 2017;16(6):9431-9440.

36. Chung $\mathrm{Y}-\mathrm{H}$, Huang $\mathrm{Y}-\mathrm{H}$, Chu $\mathrm{T}-\mathrm{H}$, et al. BMP-2 restoration aids in recovery from liver fibrosis by attenuating $\mathrm{TGF}-\beta 1$ signaling. $L a b$ Invest. 2018;98(8):999-1013.

37. Peng Q, Chen B, Wang $\mathrm{H}$, et al. Bone morphogenetic protein 4 (BMP4) alleviates hepatic steatosis by increasing hepatic lipid turnover and inhibiting the mTORC1 signaling axis in hepatocytes. Aging. 2019; 11(23):11520-11540.

38. Lytle KA, Wong CP, Jump DB. Docosahexaenoic acid blocks progression of western diet-induced nonalcoholic steatohepatitis in obese Ldlr-/mice. Plos One. 2017;12(4):e0173376.

39. Ji D, Li B, Shao Q, et al. MiR-22 Suppresses BMP7 in the Development of Cirrhosis. Cell Physiol Biochem. 2015;36(3):1026-1036.

40. Pan LX, Li LY, Zhou H, et al. TMEM100 mediates inflammatory cytokines secretion in hepatic stellate cells and its mechanism research. Toxicol Lett. 2019;317:82-91. 